\title{
An Efficient Iterative Scheme Using Family of Chebyshev's Operations
}

\author{
Seyed Hossein Mahdavi and Hashim Abdul Razak \\ StruHMRS Group, Department of Civil Engineering, University of Malaya, 50603 Kuala Lumpur, Malaysia \\ Correspondence should be addressed to Hashim Abdul Razak; hashim@um.edu.my
}

Received 11 October 2014; Revised 18 January 2015; Accepted 19 January 2015

Academic Editor: Roman Lewandowski

Copyright ( $) 2015$ S. H. Mahdavi and H. Abdul Razak. This is an open access article distributed under the Creative Commons Attribution License, which permits unrestricted use, distribution, and reproduction in any medium, provided the original work is properly cited.

\begin{abstract}
This paper presents an efficient iterative method originated from the family of Chebyshev's operations for the solution of nonlinear problems. For this aim, the product operation matrix of integration is presented, and therefore the operation of derivative is developed by using Chebyshev wavelet functions of the first and second kind, initially. Later, Chebyshev's iterative method is improved by approximation of the first and second derivatives. The analysis of convergence demonstrates that the method is at least fourth-order convergent. The effectiveness of the proposed scheme is numerically and practically evaluated. It is concluded that it requires the less number of iterations and lies on the best performance of the proposed method, especially for highly varying nonlinear problems.
\end{abstract}

\section{Introduction}

In general, one of the most popular and practical methods for the solution of nonlinear equations is iterative type schemes. The underlying concept is to linearize equations by evaluation of the nonlinear terms with known solution from the former iteration. Recently, the evaluation of iterative methods has attracted much attention due to their computational efficiency and feasibility in different disciplines of science and engineering. Fundamentally, these methods are developed by using Taylor series and algebraic decompositions. There are many studies conducted for improving the classical Newton's method by introducing predictor-corrector procedures. For example, the family of Chebyshev's methods is developed to constitute the fifth and sixth order iterative method [17]. The major drawback of offered schemes is that, for corrector step of these methods, computation of the second derivative is necessary, which most of the time is excessively difficult. There have been many attempts in the literature to overcome aforementioned shortcoming for improving these methods by making relevant algorithms free from the second derivatives [3-5]. It is shown that the rate of convergence of the modified iterative methods varies according to operation of various parameters $[6,7]$.

Mathematically, orthogonal polynomials are widely employed for functional interpolation and approximation. The basic principle lies on interpolating a function by using prescribed fix points (known as knots). Basically, it is a bad idea to increase the number of inside knots in order to get accurate results (for the higher degree interpolations); however, implementation of the superior Chebyshev functions is only option for higher degree approximations (in referring to the Runge phenomenon) $[5,8]$. In practice, functional approximation using orthogonal polynomials has been received considerable attention in dealing with solution of partial, ordinary, or fractional differential equations [9]. The main property of such series is that it converts these problems to those of solving a system of linear algebraic equations, where the repeated and redundant calculations are neglected during the process of analysis, thus making the very easy solution procedure. One of the powerful tools constructed by developed versions of orthogonal polynomials is established as wavelet functions. Considerably, the effective characteristics of wavelets such 
as localization properties and multiresolution analysis have been the focus of considerations among researchers. There are several studies available for application of this powerful tool in dynamical problems $[10,11]$ and integral equations $[12,13]$; however, there are less reports received in the literature for development of iterative methods.

Subsequently, the main contribution of this paper is to develop an operation of derivative stemmed from the particular properties of Chebyshev wavelets capable of the modification of Chebyshev's iterative methods. The structure of this paper is organized as follows. In the next section, the background of Chebyshev operations as well as the first and second kind of Chebyshev polynomials, the construction of corresponding wavelet functions, and the product matrix of integration and its well-known iterative method are briefly summarized. In Section 3 of this paper, the operation of derivative of Chebyshev is improved to approximate the first and the second derivatives. Section 4 is devoted to the proposed iterative method and analysis of convergence of the least scale of wavelet functions. For this purpose, the applicability and feasibility of the proposed method are investigated on two nonlinear scalar functions. Finally, two practical examples are given to validate and demonstrate efficiency and capability of the proposed method with emphasis on the solution of the geometrically nonlinear systems and highly varying nonlinear problems.

\section{A Brief Background of Family of Chebyshev Operations}

In the following section, a brief background of Chebyshev wavelets of the first and second kind is presented. Basically, a family of continues wavelet functions is constructed from transformation of stretched and compressed variants of the mother wavelet $\psi(x)$ defining as follows [14]:

$$
\psi_{a, b}(\tau)=|a|^{-0.5} \psi\left(\frac{\tau-b}{a}\right), \quad a, b \in \mathfrak{R}, a \neq 0
$$

In this definition, $a$ and $b$ represent the transition and scale of the mother wavelet $\psi(x)$. In general, wavelets those structured with different orders of polynomials (i.e., Chebyshev polynomials) have four underlying arguments of $\psi_{a, b}=\psi(k, n, m, \tau)$. Any positive integer $k$ is the parameter of transition, $n$ indicates the corresponding scale, $m$ is the degree (order) of the relevant polynomials, and $\tau$ denotes the local coordinate of the wavelet. Fundamentally, a function is approximated by transition of scaled wavelets on the global interval of $x_{i}$ to $x_{i+1}(i=0,1,2, \ldots)$. This global interval is dividing into the many subdivisions according to the degree of the corresponding wavelet. The idea of discretizing the global domain into the multiple partitions appropriate to the global-scaled-frequency analysis is known as Segmentation Method (SM) [10]. The main purpose of SM is to define several collocation points on the main setting domain (global points of $x_{i}$ along the global domain) and therefore to convey components of those to the new alternative domain of the analysis (i.e., local points $\tau_{i}$ in frequency domain). In this study, $2^{k-1} M$ is assumed as the number of partitions in each global interval (in referring to the SM collocation points) and the corresponding wavelets are constructed by $m=0,1,2, \ldots,\left(2^{k-1} M / 2^{k-1}\right)-1$ order of the considered polynomials. Accordingly, local coordinates of $\tau_{i}$ are defined based on SM as follows:

$$
\tau_{i}=\left(\frac{1}{2^{k-1} M}\right)(i-0.5), \quad i=1,2,3, \ldots, 2^{k-1} M .
$$

Clearly, for mapping the coordinates of interval $[0,1]$ to $\left[x_{i}, x_{i+1}\right]$ one may use $x_{2 M}=x_{i}+\tau_{i}\left(x_{i+1}-x_{i}\right)$. It should be noted that, in this study, $k=2$ is assumed for all derivations and calculations.

2.1. The First Kind of Chebyshev Wavelet. The families of orthogonal Chebyshev polynomials are classified into two main types. The first kind of Chebyshev polynomials $T_{m}(x)$ is obtained by a recursive formulation as follows $[5,15,16]$ :

$$
\begin{gathered}
T_{0}(x)=1, \quad T_{1}(x)=x, \\
T_{m+1}(x)=2 x T_{m}(x)-T_{m-1}(x), \quad m=1,2, \ldots,
\end{gathered}
$$

where the orthogonality of $T_{m}(x)$ is satisfied with respect to the weight function of $\omega(x)=1 / \sqrt{1-x^{2}}$ on $|x|<1$. Subsequently, Chebyshev wavelets of the first kind (FCW) are constructed by substituting $T_{m}(x)$ in $(1)$ as $[14,16]$

$$
\begin{aligned}
& \psi_{n, m}(x) \\
& = \begin{cases}\left(2^{k / 2}\right) \cdot \widetilde{T}_{m}\left(2^{k} x-2 n+1\right), & \frac{n-1}{2^{k-1}} \leq x<\frac{n}{2^{k-1}} \\
0, & \text { Otherwise, }\end{cases}
\end{aligned}
$$

where

$$
\widetilde{T}_{m}(x)= \begin{cases}\frac{1}{\sqrt{\pi}}, & m=0, \\ \sqrt{\frac{2}{\pi}} T_{m}(x), & m>0\end{cases}
$$

where $m=0,1,2, \ldots, M-1$ and $n=1,2, \ldots, 2^{k-1}$ represent the order of corresponding polynomials and the considered scale of the wavelet, respectively. $T_{m}(x)$ shows the recursive formula in (3) relevant to the different orders of $m$. Subsequently, dilated and transformed weight functions of $\omega(x)$ are obtained as $\omega_{n}(x)=\omega\left(2^{k} x-2 n+1\right)$ to calculate orthogonal Chebyshev wavelets of the first kind (FCW).

2.2. The Second Kind of Chebyshev Wavelet. Chebyshev polynomials of the second kind $U_{m}(x)$ are expressed by the recurrence relation of [5]

$$
\begin{gathered}
U_{0}(x)=1, \quad U_{1}(x)=2 x \\
U_{m+1}(x)=2 x U_{m}(x)-U_{m-1}(x), \quad m=1,2, \ldots .
\end{gathered}
$$

The weight functions of $\omega(x)=(2 / \pi) \sqrt{1-x^{2}},(|x|<$ 1 ) satisfy the orthogonal relation between divers degrees of 
$U_{m}(x)$. The second kind of Chebyshev wavelets (SCW) is constituted as follows [17]:

$$
\begin{aligned}
& \psi_{n, m}(x) \\
& = \begin{cases}\left(2^{k / 2}\right) \cdot \widetilde{U}_{m}\left(2^{k} x-2 n+1\right), & \frac{n-1}{2^{k-1}} \leq x<\frac{n}{2^{k-1}} \\
0, & \text { Otherwise }\end{cases}
\end{aligned}
$$

where $\widetilde{U}_{m}(x)=\sqrt{2 / \pi} U_{m}(x)$ and arguments of $k, n$, and $m$ are the same as presented before. In addition, for different orders of $m, U_{m}(x)$ is obtained from (6). Correspondingly, delayed and transitioned weight functions of $\omega_{n}(x)=\omega\left(2^{k} x-2 n+1\right)$ are developed to calculate orthogonal SCWs.

2.3. Functional Decomposition. Mathematically, any quadratically integrable function $f(x)$ may be decomposed by the truncated series of a wavelet's family (i.e., FCW or SCW) as follows $[13,14,17]$ :

$$
f(x) \cong \sum_{n=1}^{2^{k-1} M-1} \sum_{m=0} c_{n, m} \psi_{n, m}(x)=C^{T} \Psi(x)
$$

where $C$ denotes the vector of coefficients of the considered wavelets, that is, FCW or SCW. Furthermore, $\Psi(x)$ represents the vector of corresponding wavelet functions defined as

$$
\begin{aligned}
& C=\left[c_{1}, c_{2}, c_{3}, \ldots, c_{2^{k-1}}\right]_{2^{k-1} M \times 1}^{T} \\
& \Longleftrightarrow c_{i}=\left[c_{i 0}, c_{i 1}, c_{i 2}, \ldots, c_{i, M-1}\right]^{T}, \\
& i=1,2, \ldots, 2^{k-1}, \\
& \Psi(x)=\left[\psi_{1}, \psi_{2}, \psi_{3}, \ldots, \psi_{2^{k-1}}\right]_{2^{k-1} M \times 1}^{T} \\
& \Longleftrightarrow \psi_{i}(x)=\left[\psi_{i 0}(x), \psi_{i 1}(x), \psi_{i 2}(x), \ldots, \psi_{i, M-1}(x)\right]^{T} .
\end{aligned}
$$

Eventually, a $2^{k-1} M \times 2^{k-1} M$-dimensional matrix of $\phi_{n, m}(x)$ is developed as

$$
\phi_{n, m}(x)=\left[\begin{array}{lllll}
\Psi\left(x_{1}\right) & \Psi\left(x_{2}\right) & \ldots & \Psi\left(x_{i}\right)
\end{array}\right]_{2^{k-1} M \times 2^{k-1} M} .
$$

The square matrix of $\phi_{n, m}(x)$ is populated with vectors of wavelet functions for a set of discrete SM local points $\left(x_{i}, i=\right.$ $\left.1,2,3, \ldots, 2^{k-1} M\right)$.

2.4. Product Matrix of Integration. The product matrix $P$ of integration (for FCW and SCW) is briefly discussed in this section. The detailed calculations of $P$ can be found in $[14,17]$. Assumption of the integration of $\Psi(x)$ is as follows (for $k=$ 2):

$$
\int_{0}^{1} \Psi_{2 M}(x) d t=P_{2 M} \Psi(x)
$$

In (11), the subscripts of $\Psi_{2 M}$ and $P_{2 M}$ denote the dimension of matrices. Accordingly, the $2^{k} M \times 2^{k} M$-dimensional operational matrix $P$ for FCW and SCW is derived as

$$
P=\frac{1}{2^{k}}\left[\begin{array}{ccccc}
{[L]_{M \times M}} & {[F]_{M \times M}} & F & \ldots & F \\
{[O]_{M \times M}} & {[L]_{M \times M}} & F & \ldots & F \\
O & O & \ddots & \ddots & \vdots \\
\vdots & \vdots & \ldots & \ddots & F \\
O & O & \cdots & O & L
\end{array}\right]
$$

where $M \times M$ square matrices $F$ and $L$ are given as follows:

$$
F=\left[\begin{array}{cccccc}
2 & 0 & 0 & \cdots & 0 & 0 \\
0 & 0 & 0 & \cdots & 0 & 0 \\
a_{1} & 0 & 0 & \cdots & 0 & 0 \\
0 & 0 & 0 & \ddots & \vdots & \vdots \\
\vdots & \vdots & \vdots & \vdots & \ddots & \vdots \\
a_{2} & 0 & \cdots & \cdots & 0 & 0
\end{array}\right],
$$

$$
L=\left[\begin{array}{cccccccc}
1 & a_{6} & a_{9} & a_{12} & \cdots & 0 & 0 & 0 \\
a_{3} & 0 & a_{10} & a_{13} & \cdots & 0 & 0 & 0 \\
a_{4} & a_{7} & 0 & a_{14} & \cdots & 0 & 0 & 0 \\
a_{5} & a_{8} & a_{11} & 0 & \cdots & \vdots & \vdots & \vdots \\
\vdots & \vdots & \vdots & \vdots & \ddots & \vdots & \vdots & \vdots \\
\vdots & \vdots & \vdots & \vdots & \vdots & \ddots & \vdots & \vdots \\
a_{15} & 0 & 0 & \cdots & \cdots & a_{17} & \ddots & a_{19} \\
a_{16} & 0 & 0 & \cdots & \cdots & 0 & a_{18} & 0
\end{array}\right] .
$$

It is deduced from (12) and (13) that the population of arrays of $P$ is similar for FCW and SCW. In detail, coefficients of $a_{i}$ are defined for FCW and SCW and tabulated in Table 1 $[14,17]$.

It is to be pointed out that $M$ refers to $2^{k-1} M / 2^{k-1}$. To calculate product matrix of $P$ for FCW and SCW, a backward algorithm of program coding is recommended. In other words, only the first four rows and columns of $P$ are being computed, initially. Then, the elements of $P$ being calculated from the last row and column $M$ th until computation of the 5th row and column. To clarify the expression of wavelet's parameters of FCW and SCW, matrices of $\phi_{4,4}$ and $P_{4,4}$ are calculated and tabulated in Table 2 for $k=2, M=2$.

The practical effectiveness of data displayed in Table 2 will be discussed in detail later.

2.5. The Iterative Chebyshev's Operation. The basic method to solve nonlinear problems is the classical Newton's method. 
TABLE 1: Coefficients of $a_{i}$, defined in (13) corresponding to FCW and SCW.

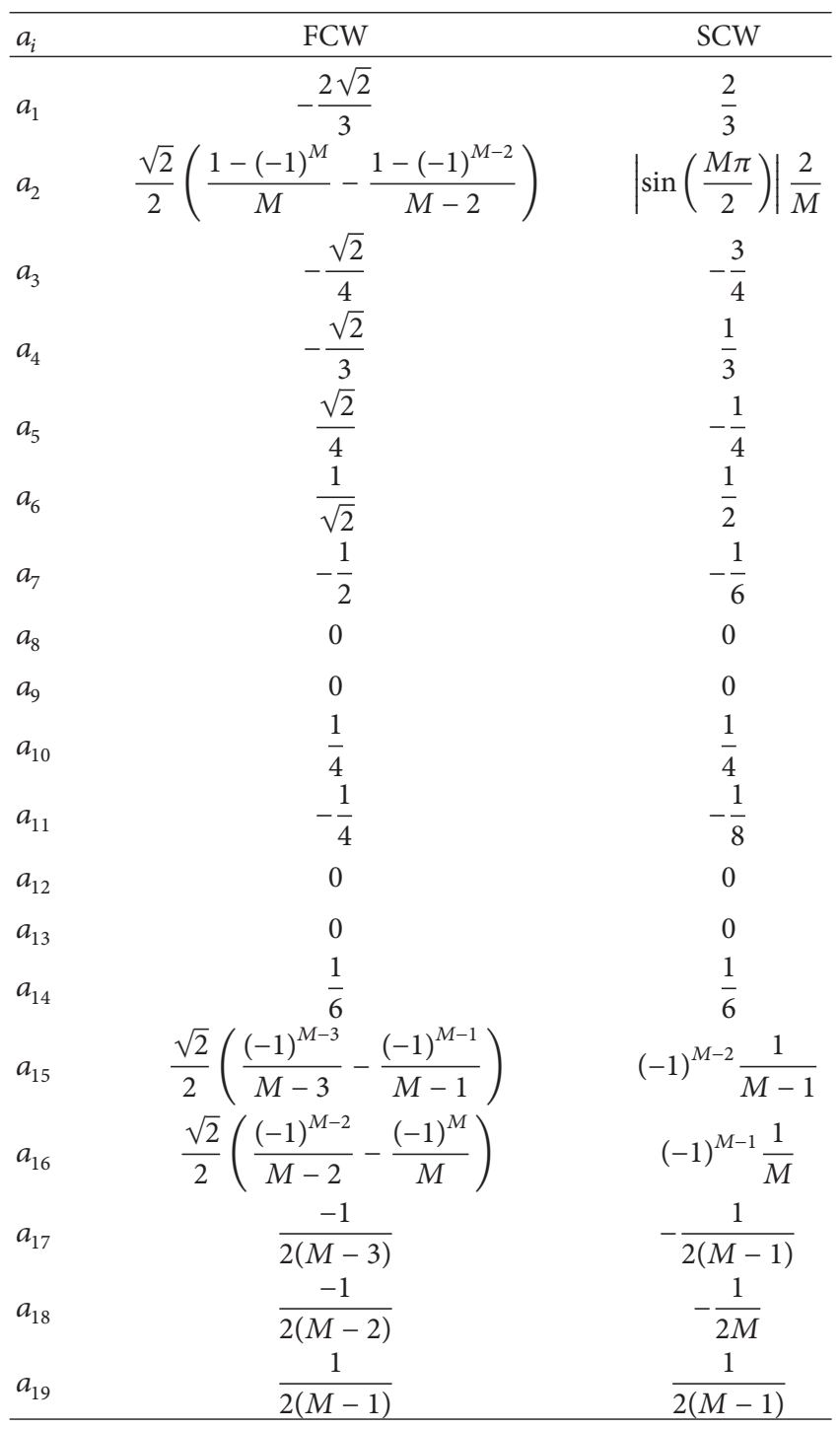

This scheme converges quadratically and for a single nonlinear equation is expressed as $x_{n+1}=x_{n}-f\left(x_{n}\right) / f^{\prime}\left(x_{n}\right)$ [2$4]$. One of the iterative methods utilized to improve Newton's scheme lies on the third-order convergent Chebyshev's method. According to this algorithm, the new point of $x_{n+1}$ is iterated from the preceding point of $x_{n}$ by [7]

$$
x_{n+1}=x_{n}-\left(1+\frac{1}{2} \frac{f^{\prime \prime}\left(x_{n}\right) f\left(x_{n}\right)}{f^{\prime}\left(x_{n}\right)}\right) \frac{f\left(x_{n}\right)}{f^{\prime}\left(x_{n}\right)} .
$$

As it is shown in (14), iterative Chebyshev's algorithm is rigorously dependent on the calculation of second derivatives, in which most of the time it is neither possible nor practical. Thus, the computing process is excessively difficult, and therefore the practical applications are extremely restricted, particularly, for solving highly varying nonlinear systems. Consequently, the main contribution of our study is discovered here, whereby an iterative procedure is proposed to improve (14) by employing FCW and SCW free from computation of the second derivatives.

\section{The Proposed Method for Operation of Derivative}

In this section, an efficient approach is proposed for approximation of derivatives using free-scaled wavelet functions. The proposed method is applicable for various wavelet basis functions, once the product matrix of integration and wavelet coefficient vectors are available. For this purpose, integral functions are numerically developed from local coordinates to global system. For a differentiable function of $f(x): x \in$ $\left[x_{n}, x_{n+1}\right]$, indefinite formulation of (15) is considered based on the Newton theorem as follows:

$$
f(x)=f\left(x_{n}\right)+\int_{x_{n}}^{x} f^{\prime}(x) d x .
$$

We initialize $x_{n+1}=x_{n}-f\left(x_{n}\right) / f^{\prime}\left(x_{n}\right)$ for definite form of (15); let $d_{n}=x_{n+1}-x_{n}$. Using (8) the derivative function is approximated on global coordinates as follows:

$$
f^{\prime}(x)=C^{T} \cdot \Psi(x) .
$$

Substituting into (15) we have

$$
f\left(x_{n+1}\right)=f\left(x_{n}\right)+\int_{x_{n}}^{x_{n+1}} C^{T} \cdot \Psi(x) d x .
$$

Multiplying by operational matrix $P$ of integration, adding initial constant of integration, we have

$$
f\left(x_{n+1}\right)=f\left(x_{n}\right)+d_{n} \cdot C^{T} \cdot P \cdot \Psi(x)+f^{\prime}\left(x_{0}\right),
$$

where $d_{n}$ is operated for mapping local characteristics of Chebyshev wavelet to global ones. To simplify this equation, constant quantities are approximated by Chebyshev wavelets in each step. For this purpose, the unity is being expanded by the Chebyshev wavelet as $[10,15,16]$

$$
1 \cong I^{*} \Psi(t) \cong D\left[1_{1,1}, 0,0, \ldots, 1_{1, M+1}, 0,0, \ldots\right] \Psi(t),
$$

where $D=\sqrt{\pi / 4}$ and $\sqrt{\pi / 8}$ for FCW and SCW, respectively. Therefore, initial values are developed as $2^{k-1} M \times 1$ dimensional vectors corresponding to collocation points:

$$
\begin{gathered}
f^{\prime}\left(x_{0}\right)=f^{\prime}\left(x_{0}\right) I^{*} \Psi(x), \\
f\left(x_{n+1}\right)=f\left(x_{n+1}\right) I^{*} \Psi(x), \\
f\left(x_{n}\right)=f\left(x_{n}\right) I^{*} \Psi(x) .
\end{gathered}
$$

Substituting (20) into (18) we have

$$
\begin{aligned}
f\left(x_{n+1}\right) I^{*} \Psi(x)= & f\left(x_{n}\right) I^{*} \Psi(x)+d_{n} \cdot C^{T} \cdot P \\
& \cdot \Psi(x)+f^{\prime}\left(x_{0}\right) I^{*} \Psi(x) .
\end{aligned}
$$

Subsequently, eliminating $\Psi(x)$ from both sides of (21) and after algebraic calculations $C^{T}$ is being calculated. Using 
TABLE 2: Corresponding components of wavelets $\phi_{i, j}$ and $P$, calculated on four SM $=4$ points for FCW and SCW.

\begin{tabular}{|c|c|c|c|c|c|c|c|c|c|}
\hline \multirow{2}{*}{\multicolumn{2}{|c|}{$\begin{array}{l}\text { Column } j \\
\text { Row } i\end{array}$}} & \multicolumn{2}{|c|}{1} & \multicolumn{2}{|c|}{2} & \multicolumn{2}{|c|}{3} & \multicolumn{2}{|c|}{4} \\
\hline & & $\phi_{i, j}$ & $P_{i, j}$ & $\phi_{i, j}$ & $P_{i, j}$ & $\phi_{i, j}$ & $P_{i, j}$ & $\phi_{i, j}$ & $P_{i, j}$ \\
\hline \multirow{2}{*}{1} & FCW & 1.1284 & 0.2500 & 1.1284 & 0.1768 & 0 & 0.5000 & 0 & 0 \\
\hline & SCW & 1.5958 & 0.2500 & 1.5958 & 0.1250 & 0 & 0.5000 & 0 & 0 \\
\hline \multirow{2}{*}{2} & FCW & -0.7979 & -0.0884 & 0.7979 & 0 & 0 & 0 & 0 & 0 \\
\hline & SCW & -1.5958 & -0.1875 & 1.5958 & 0 & 0 & 0 & 0 & 0 \\
\hline \multirow{2}{*}{3} & FCW & 0 & 0 & 0 & 0 & 1.1284 & 0.2500 & 1.1284 & 0.1768 \\
\hline & SCW & 0 & 0 & 0 & 0 & 1.5958 & 0.2500 & 1.5958 & 0.1250 \\
\hline \multirow{2}{*}{4} & FCW & 0 & 0 & 0 & 0 & -0.7979 & -0.0884 & 0.7979 & 0 \\
\hline & SCW & 0 & 0 & 0 & 0 & -1.5958 & -0.1875 & 1.5958 & 0 \\
\hline
\end{tabular}

Note: FCW = first Chebyshev wavelet, SCW = second Chebyshev wavelet.

(16), $f^{\prime}(x)$ is approximated on $2 M$ global points. The same approach is employed on $f^{\prime}(x)$ to compute the second derivative of $f^{\prime \prime}(x)$. The proposed method is implemented on $f(x)=\sin \left(x^{2}\right) ; x \in[0,2]$, in which its definite $f^{\prime}(x)=2 x$. $\cos \left(x^{2}\right)$ and $f^{\prime \prime}(x)=2\left[\cos \left(x^{2}\right)-2 x^{2} \sin \left(x^{2}\right)\right]$ exist. The first and second derivatives are calculated for $2 M=8$ collocation points of FCW and SCW and have been compared with exact values (designated by original $d f / d x$ or $d(d f / d x)$ ) in Figures 1 and 2, respectively. The approximated results for the first and second derivatives of $f(x)$ are designated in figures by $\operatorname{App}(d f / d x)$ and $\operatorname{App}(d(d f / d x))$, respectively.

The schematic view of results in Figures 1 and 2 lies on the better accuracy of SCW, when $2 M=8$ is applied. For the purpose of a detailed comparison, various $2 M$ points are employed through the proposed method corresponding to diverse scales of FCW and SCW to calculate the first and second derivative of considered $f(x)$. The comparison of results is depicted in Figures 3 and 4 related to the FCW and SCW, respectively. Accordingly, the percentile total average error (PTAE) measurement is presented for the purpose of comparison. By assumption of $\alpha$ for the closed-form solution and $X_{c}$ as the calculated value, PTAE is defined as PTAE $=$ $100 \sum\left|\left(\alpha-X_{c}\right) / \alpha\right|$.

The measured PTAE data shown in Figures 3 and 4 illustrate that free scales of SCW approximate the first and second derivatives more accurate than those of FCW. For instance, PTAE $=89.49 \%$ is measured for the second scale of FCW, while this value is considerably decreased to $9.32 \%$ for the same scale of SCW. As it is shown in Figure 3, the accuracy of the second derivative is more than the first one. This is because the oscillatory shape of the results coincides with the exact result for the second derivative, in contrast to the calculated results by SCW for the first derivative. Significantly, the error measurement of the proposed method using higher scales of SCW demonstrates the superiority of this wavelet. Finally, it is apparent from the figures that end point errors diversely affect the accuracy of results for the higher order approximations shown for $2 M=64$ collocation points.

In addition, the proposed scheme is applicable in problems with several unknowns, where the tangent line becomes a tangent (hyper) plane. For instance, (15) is developed for a function of two variables $f(x, y)$ as follows:

$$
f\left(x, y=y_{0}\right)=f\left(x_{n}, y=y_{0}\right)+\int_{x_{n}}^{x} f_{x}^{\prime}\left(x, y=y_{0}\right) d x,
$$

where $x$ and $y=y_{0}$ represent the first variable and the constant point for the second variable, respectively. $f_{x}^{\prime}\left(x, y=y_{0}\right)$ indicates the derivative of $f(x, y)$ with respect to $x$ while $y=y_{0}$ and the subscripts $x$ and $y$ are changed for the next variable as

$$
f\left(x=x_{0}, y\right)=f\left(x=x_{0}, y_{n}\right)+\int_{y_{n}}^{y} f_{y}^{\prime}\left(x=x_{0}, y_{n}\right) d y \text {. }
$$

Consequently, $f_{x}^{\prime}$ and $f_{y}^{\prime}$ are calculated, and therefore the normal vector $(\vec{n})$ to the plane at $x_{0}$ and $y_{0}$ is derived as

$$
\vec{n}=\left\langle f_{x}^{\prime}\left(x_{0}, y_{0}\right), f_{y}^{\prime}\left(x_{0}, y_{0}\right),-1\right\rangle
$$

\section{The Proposed Iterative Method}

The proposed iterative method contains the set of modified predictors-correctors explained in (14). The operation of derivative proposed in previous section is employed to approximate the second derivatives using FCW and SCW. Basically, the most accurate results are computed for the approximation of only the second derivative; however, the method is also applicable for approximation of the first derivative. For this purpose, $y_{n}=x_{n}-f\left(x_{n}\right) / f^{\prime}\left(x_{n}\right)$ is initially predicted. Later, the second derivatives of $f(x)$ for $x \in$ $\left[x_{n}, y_{n}\right]$ are approximated using FCW or SCW on $2 M$ collocation points. Eventually, the new point $x_{n+1}$ is iterated using Chebyshev's iterative algorithm of (14). The geometric construction of the proposed method is illustrated in Figure 5. Accordingly, the analysis of convergence of the proposed method using SCW or FCW may be satisfied for the least orders of $2 M=2$ corresponding to the first column and 


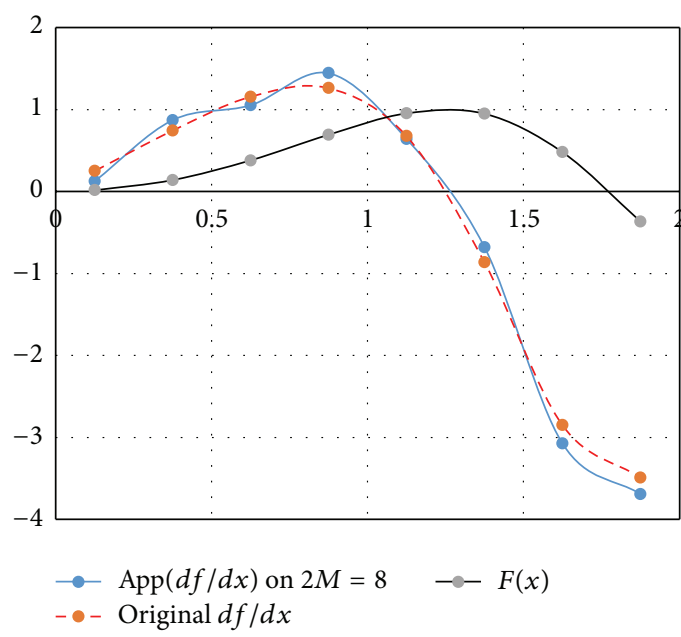

(a)

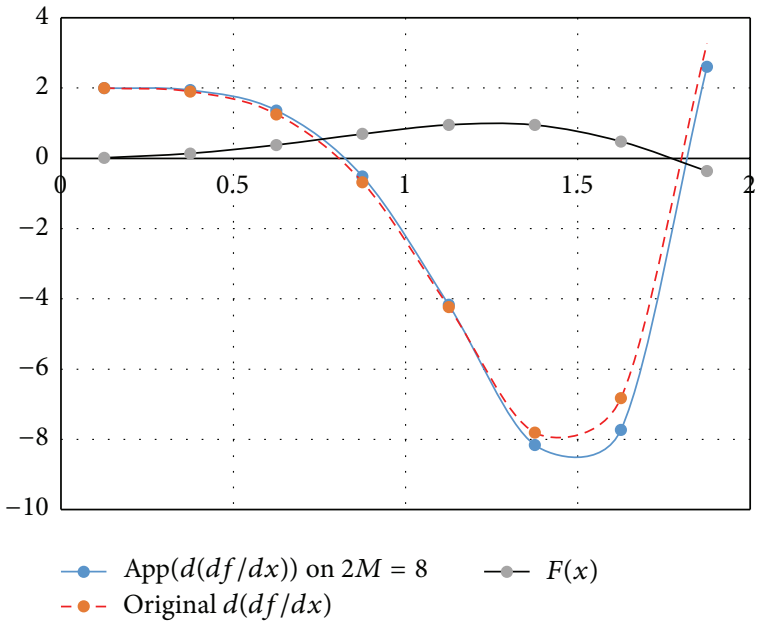

(b)

FIGURE 1: The approximated results using the proposed operation of derivative of FCW on $2 M=8$ collocation points for calculation of (a) the first and (b) the second derivative.

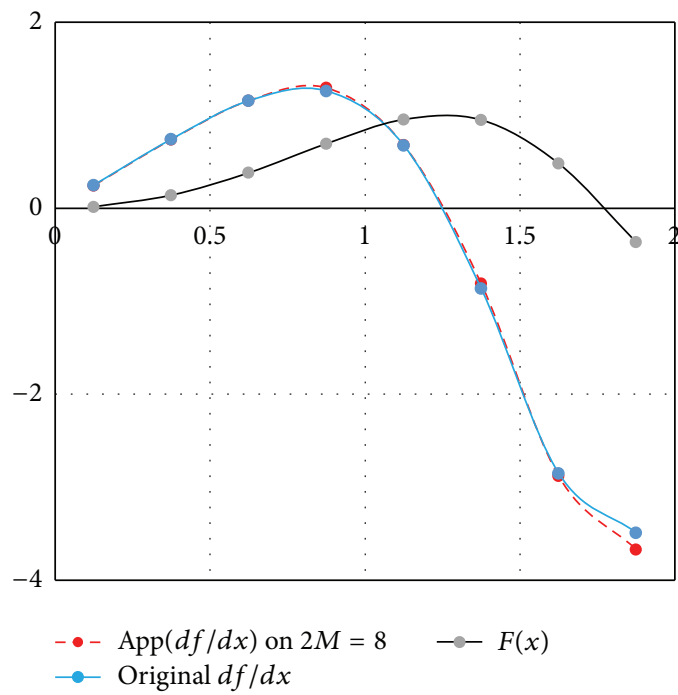

(a)

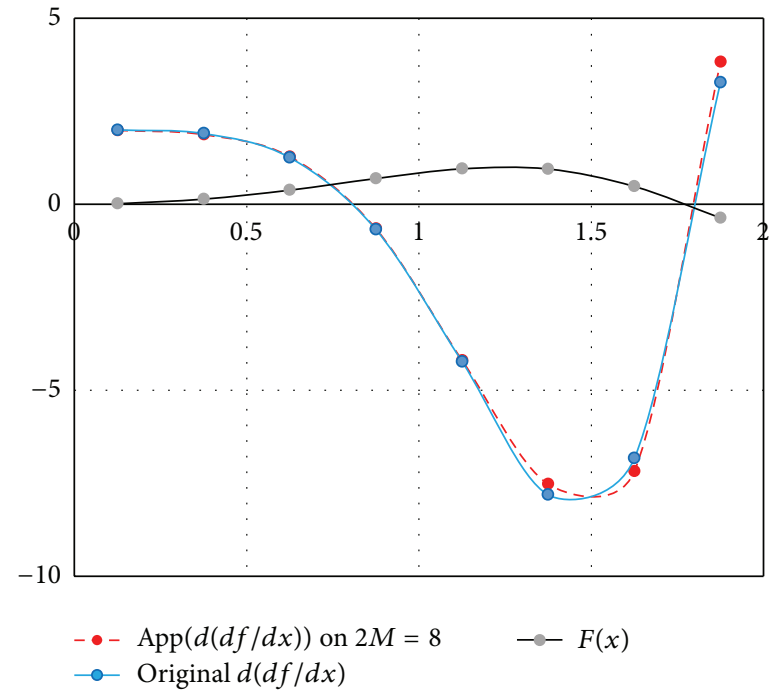

(b)

FIGURE 2: The approximated results using the proposed operation of derivative of SCW on $2 M=8$ collocation points for computation of (a) the first and (b) the second derivative.

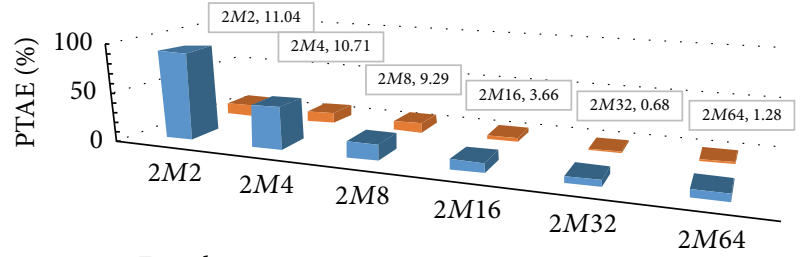

- First derivative

- Second derivative

Figure 3: PTAE measurement corresponding to different scales (2M collocations) of FCW.

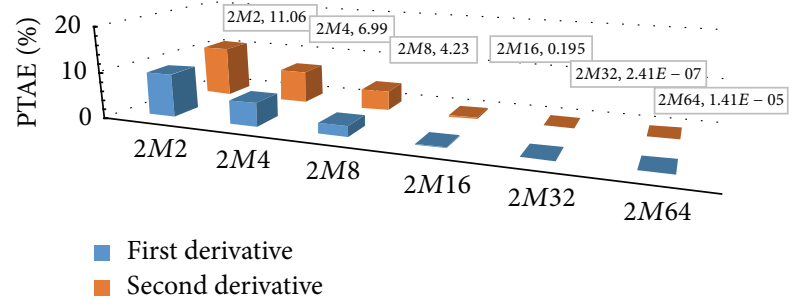

FIGURE 4: PTAE measurement corresponding to various scales (2M collocations) of SCW. 
row of matrix $P$ and vector $C$ as (the matrix calculus is then satisfied):

$$
x_{n+1}=x_{n}-\left(1+\frac{1}{2} \frac{C^{T} \psi(x) \cdot C^{T} P^{2} \psi(x)}{C^{T} P \psi(x)}\right) \frac{f\left(x_{n}\right)}{f^{\prime}\left(x_{n}\right)} .
$$

Using $(7)$ and substituting $\psi_{1,1}(x)=(\sqrt{2 / \pi})(16 x-4)$ for SCW yield

$$
x_{n+1}=x_{n}-\left(1+\frac{1}{2} \eta\left(16 x_{n}-4\right)\right) \frac{f\left(x_{n}\right)}{f^{\prime}\left(x_{n}\right)} \text {, }
$$

where $\eta$ is assumed as an independent variable of the error function. On the other hand, let $\alpha$ be a simple root of $f$; thus $f(\alpha)=0$ and $f^{\prime}(\alpha) \neq 0$. As long as $f$ is a sufficiently differentiable function, using Taylor series expansion of $f$ and $f^{\prime}$ around $x_{n}=\alpha$ gives $[1-4,8]$

$$
\begin{aligned}
f\left(x_{n}\right)= & f^{\prime}(\alpha)\left(x_{n}-\alpha\right)+\frac{1}{2 !} f^{\prime \prime}(\alpha)\left(x_{n}-\alpha\right)^{2} \\
& +\frac{1}{3 !} f^{\prime \prime \prime}(\alpha)\left(x_{n}-\alpha\right)^{3}+\cdots+O\left(\left(x_{n}-\alpha\right)^{4}\right) .
\end{aligned}
$$

Using $O\left(e_{n}{ }^{l}\right)=\sum_{i=l}^{\infty} c_{i} e_{n}{ }^{i}$ and $c_{s}=(1 / s !)\left(f^{s}(\alpha) / f^{\prime}(\alpha)\right), s=$ $1,2, \ldots$ and $e_{n}=x_{n}-\alpha,(27)$ is therefore developed as follows:

$$
\begin{aligned}
f\left(x_{n}\right)=f^{\prime}(\alpha)\left[e_{n}+c_{2} e_{n}{ }^{2}+c_{3} e_{n}{ }^{3}\right. & \\
& \left.+c_{4} e_{n}{ }^{4}+c_{5} e_{n}{ }^{5}+\cdots+O\left(e_{n}{ }^{6}\right)\right] .
\end{aligned}
$$

Accordingly, the first derivative of $f$ is obtained as

$$
\begin{aligned}
f^{\prime}\left(x_{n}\right)=f^{\prime}(\alpha)[1 & +2 c_{2} e_{n}+3 c_{3} e_{n}{ }^{2} \\
& \left.+4 c_{4} e_{n}{ }^{3}+5 c_{5} e_{n}{ }^{4}+\cdots+O\left(e_{n}{ }^{5}\right)\right] .
\end{aligned}
$$

Dividing (28) by (29), one may obtain

$$
\frac{f\left(x_{n}\right)}{f^{\prime}\left(x_{n}\right)}=e_{n}-c_{2} e_{n}^{2}+2\left(c_{2}^{2}-c_{3}\right) e_{n}^{3}+\cdots+O\left(e_{n}^{4}\right) \text {. }
$$

Let $e_{n}=x_{n}-\alpha$; therefore, (26) is developed as follows:

$$
\begin{aligned}
x_{n+1}= & x_{n}-\left(1+\frac{1}{2} \eta\left(16 e_{n}+16 \alpha-4\right)\right) \\
& \cdot\left[e_{n}-c_{2} e_{n}{ }^{2}+2\left(c_{2}^{2}-c_{3}\right) e_{n}^{3}+\cdots+O\left(e_{n}^{4}\right)\right] .
\end{aligned}
$$

TABLE 3: Comparison of the proposed iterative methods and Newton's method for solution of $f_{1}$ and $f_{2}$.

\begin{tabular}{ccccccc}
\hline$f\left(x_{n}\right)$ & $x_{0}$ & Newton & FCW (2M8) & D\% & SCW (2M8) & D\% \\
\hline \multirow{2}{*}{$f_{1}$} & 0 & 4 & 2 & $50 \%$ & 2 & $50 \%$ \\
& 0.5 & 4 & 2 & $50 \%$ & 2 & $50 \%$ \\
\hline \multirow{2}{*}{$f_{2}$} & 1.2 & 6 & 2 & $66.7 \%$ & 2 & $66.7 \%$ \\
& 3.5 & 7 & 4 & $42.85 \%$ & 3 & $57.14 \%$ \\
\hline
\end{tabular}

Note: FCW (2M8), SCW $(2 M 8)=$ first and second kind of Chebyshev wavelets on $2 M=8$.

This means that the order of convergence of the proposed method is at least four $\left(e_{n}{ }^{4}\right)$, when the least scale of SCW is employed. The same approach is applied for FCW and the same convergence rate is obtained.

Now we present two examples to investigate the efficiency of the improved iterative method. The solution of two scalar nonlinear equations $f_{1}(x)$ and $f_{2}(x)$ is considered as follows:

$$
\begin{aligned}
& f_{1}(x)=x^{2}-e^{x}-3 x+2, \quad \alpha=0.2575302854 \\
& f_{2}(x)=\sin ^{2}(x)-x^{2}+1, \quad \alpha=1.4044916482
\end{aligned}
$$

where $\alpha$ denotes the simple root of the nonlinear equation and the prescribed error measurement at the ending of the iteration process is $\left|f\left(x_{n}\right)\right|<1 \cdot e-6$. The total number of iterations (NIt) and relative percentile reduction of NIt (designated by $D \%$ ) from the starting point of $x_{n}$ are compared for the classical Newton's method and the proposed methods of FCW and SCW (for $2 M=8$ ), shown in Table 3.

Obviously, data displayed in Table 3 demonstrate that FCW and SCW require lesser number of iterations than Newton's method. In addition, it shows the satisfactory performance of the developed method using $2 M=8$ scales of FCW, while the superior performance is observed for SCW.

\section{Numerical Application}

One of the important practices of iterative methods is nonlinear structural analysis. In structural analysis, $\{f(x)\}=$ $P$ is the equilibrium equation of structural elements with the vector of nodal internal loadings $\{f\}$ subjected to external forces $P$. In a nonlinear structure, the load-deformation relations of each degree of freedom (DOF) illustrate that $\{f\}$ is a nonlinear function of displacements $\{x\}$. Therefore, the convenient analysis will be carried out by using the incremental form of structural equilibrium of $\left[K_{t}\right]\{\Delta x\}=$ $\{\Delta P\}$. In this description, $\left[K_{t}\right]$ is the gradient matrix of the equations; namely, tangential stiffness matrix contains both the linear stiffness matrix $\left[K_{L}\right]$ and the geometric stiffness matrix of $\left[K_{G}\right]$ stemmed from updated stresses due to the deformations. This matrix is predicted at the first step of each increment and therefore is corrected, iteratively ( $\left[K_{t}^{\text {Predicted }}\right]$ and $\left[K_{t}^{\text {Corrected }}\right]$ shown in Figure 5). Furthermore, the incremental load-control schemes of arclength and classical Newton-Raphson are the most popular methods to follow the nonlinear equilibrium path of loaddisplacement, however, with various performance. Figure 5 


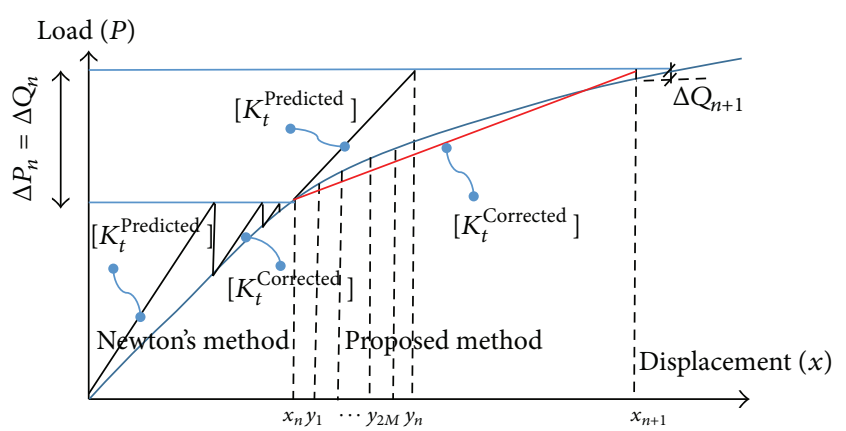

FIGURE 5: The geometric construction of the proposed method using FCW or SCW.

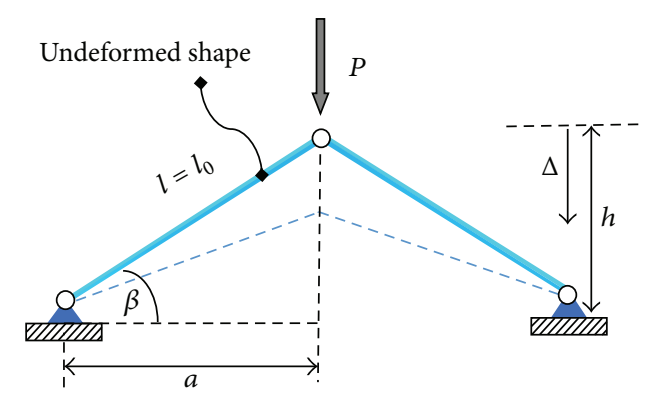

Figure 6: William's toggle frame subjected to the $P$ at the apex.

describes the overall process of Newton-Raphson method and the proposed method, whereby the out-of-balanced force $\Delta Q_{n}$ is considered for new iterations (correctors).

The geometrically nonlinear analysis of the well-known William's toggle frame is considered in this section. Figure 6 shows the geometry of this simple frame comprised of two truss elements and one DOF. $E=20.7 e 3 \mathrm{~N} / \mathrm{cm}^{2}, A=1 \mathrm{~cm}^{2}$, and $l=254 \mathrm{~cm}$ are chosen as the modulus of elasticity, crosssectional area, and undeformed length, respectively. The load increment of $\Delta P=550 \mathrm{kN}$ is applied at the top node of this truss for two increments $\left(\beta=30^{\circ}\right)$.

In this application, the displacement-control criterion is utilized to stop the ending iterations and the tolerance factor $1 e-3$ is accordingly chosen. Basically, the geometrically nonlinear equilibrium and the one DOF tangential stiffness $\left[K_{t}\right]$ of this structure (which is the gradient of the $f(x)$ ) are defined as [18]

$$
\begin{aligned}
f(x)= & 2 E A\left(\sin (\beta)-\frac{\Delta}{l}\right) \\
& \cdot\left[\frac{1}{\sqrt{1+(\Delta / l)^{2}-2(\Delta / l) \sin (\beta)}}-1\right], \\
K_{t}=1 & -\frac{\cos ^{2}(\beta)}{\left(1+(\Delta / l)^{2}-2(\Delta / l) \sin (\beta)\right)^{1.5}} .
\end{aligned}
$$

This structure is analyzed using the proposed method of FCW and SCW $(2 M=8)$ and the classical Newton's method for two increments of load. For the classical Newton's
TABLE 4: Comparison of number of iterations (NIt) for two increments of $\triangle P$.

\begin{tabular}{lccccc}
\hline & & FCW & & SCW \\
& NIt & $D \%$ & NIt & $D \%$ \\
\hline $2 M=2$ & 32 & $33 \%$ & 30 & $37 \%$ \\
$2 M=4$ & 30 & $37 \%$ & 29 & $39 \%$ \\
$2 M=8$ & 29 & $39 \%$ & 24 & $50 \%$ \\
\hline
\end{tabular}

method the convergence criteria are achieved by NIt $=48$ for two increments (predictions). Subsequently, total number of iterations (NIt) and relative percentile reductions are displayed in Table 4 corresponding to various scales of FCW and SCW.

It is apparent from Table 4 that the convergence criterion is accomplished by less iterations using the 8th scales of SCW. It is shown that this scale of wavelet rapidly converges. Furthermore, the competency of the proposed method is also confirmed by FCW, while it requires the less number of iterations compared with Newton's method. Actually, the main computational efficiency of the proposed method is not discovered well through this application, where the similar number of increments is utilized for Newton's and the proposed method. As a result, the computational time (recorded with a same hardware) due to the 8th scales of the proposed method reached the highest value of $0.5 \mathrm{sec}$ compared with the minimum value of $0.01 \mathrm{sec}$ recorded for the solution of Newton's method. However, the best rate of convergence is achieved by the proposed method. It is concluded that the best performance of the proposed method is revealed in statically or dynamically highly varying nonlinear problems, in which the initial value of $f_{\left(x_{n}\right)}^{\text {'Predicted }}$ may be predicted for a long increment of $\Delta P$ as shown in Figure 7.

Figure 7 shows the shortcoming of Newton's method for the highly varying nonlinear problems. In contrast, it is illustrated that the highly varying nonlinear behaviors are accurately captured using the proposed method of FCW or SCW on corresponding collocation points. In this figure $x_{2 M}^{w}$ refers to the $2 M$ collocation points of wavelet, the same as introduced in Figure 5 for initial predictions of $y_{1}$ to $y_{2 M}$. It is shown that the only increment of $\Delta P$ applied for the proposed method will be divided to at least four increments of Newton's scheme in pursuit of an accurate analysis (for this particular case). Consequently, the cost of analysis is significantly increased depends on existed nonlinearity. Such nonlinear behaviors cannot happen on our practical example (geometric nonlinearity of structures), where the nonlinear path is almost linear until the first critical point (inflection point). However in many applications of physics or chemistry aforementioned highly nonlinear characteristics are considered through both static and dynamic analysis.

To investigate the effectiveness of the proposed method in highly varying nonlinear problems, nonlinear function of 

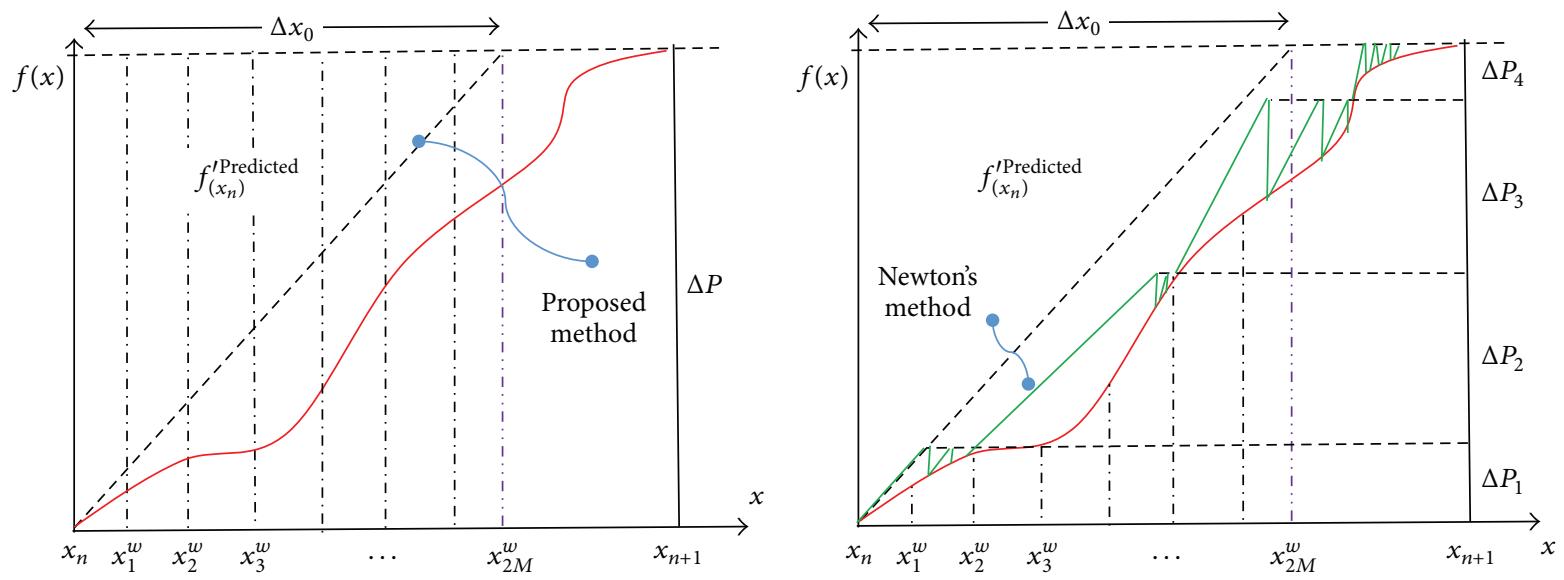

FIGURE 7: The geometric illustration of computational efficiency of the proposed method compared with Newton's method.

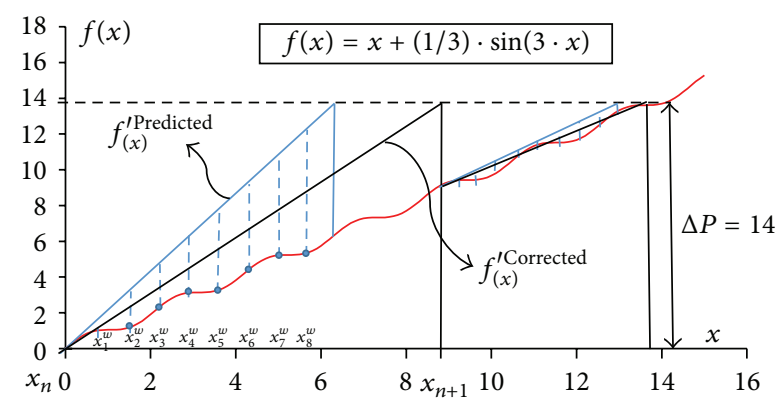

FIgURE 8: The schematic view of iterative solution of nonlinear $f(x)$ using the proposed method.

$f(x)=x+\alpha \operatorname{Sin}(\beta x), \alpha=1 / 3$, and $\beta=3$ is evaluated by Newton's scheme and various scales of FCW and SCW. For this purpose, one increment of $\Delta P=14$ is considered for the proposed procedure, whereas eight increments of $\Delta P$ are utilized for the classical Newton's method to capture details of foregoing function. The schematic view of iterative solution of $f(x)$ using the proposed scheme is shown in Figure 8.

As it is shown in Figure 8, by using the long increment of $\Delta P=14$, details of the nonlinear function are almost accurately evaluated to iterate new $x_{n+1}$ from $x_{n}$. The prescribed error measurement at the ending of the iteration process is $\left|f\left(x_{n}\right)\right|<1 \cdot e-5$. The total number of iterations (NIt), relative percentile reduction of NIt (designated by $D \%$ ) from the starting point of $x_{n}$, and computation time involved (CT) are compared in Table 5 for the proposed methods of FCW and SCW. It is to be noted that NIt $=361$ is recorded for the classical Newton's method ( 8 numbers of $\Delta P$ ) with CT = $0.53 \mathrm{sec}$.

Data in Table 5 shows that the best efficiency is recorded for SCW $(2 M=8)$ by NIt $=8$ and the least time consumption of CT $=0.19 \mathrm{sec}$. It is shown that, for the low scales of FCW and SCW $(2 M=2$ or 4$)$, because of the simplicity of function's evaluation of $2 M=2$, the final computational times are almost the same. However, for the larger scales, time
TABle 5: Comparison of number of iterations (NIt) and computational time (CT) for one increment of $\Delta P=14$.

\begin{tabular}{lcccccc}
\hline & \multicolumn{3}{c}{ FCW } & \multicolumn{3}{c}{ SCW } \\
& NIt & $D \%$ & CT (sec) & NIt & $D \%$ & CT (sec) \\
\hline $2 M=2$ & 21 & $94.1 \%$ & 0.28 & 19 & $94.7 \%$ & 0.25 \\
$2 M=4$ & 15 & $95.8 \%$ & 0.27 & 14 & $96.1 \%$ & 0.25 \\
$2 M=8$ & 10 & $97.2 \%$ & 0.22 & 8 & $97.8 \%$ & 0.19 \\
\hline
\end{tabular}

consumption is decreased due to less number of iterations. Finally, this numerical example demonstrates the capability of the proposed method in such applications by using larger scales, in which details of nonlinear problem are accurately captured prior to the new iterations.

\section{Conclusion}

This paper presented an efficient iterative approach for solving nonlinear equations. For this purpose, the operation of derivative is developed using free-scaled Chebyshev wavelet functions of the first and second kind. Accordingly, the Chebyshev iterative method is improved by calculation of the second derivatives using the proposed operations. It is shown that the rate of convergence of the proposed method using the less scales of FCW and SCW is at least four. The robustness of the proposed algorithm in terms of computational efficiency is validated for numerical examples as well as a practical example. It is deduced that the proposed method requires the less number of iterations and therefore rapidly converges, particularly, when the second kind of Chebyshev wavelet is implemented. The practical applications demonstrate the capability of the proposed method for solution of highly varying nonlinear problems. It is recommended to employ the proposed method for dynamic nonlinear analysis, where the dynamic solution is benefited by the multiresolution characteristics of wavelet functions for capturing the important details of considered systems for both geometry and material nonlinearity. 


\section{Conflict of Interests}

The authors declare that there is no conflict of interests regarding the publication of this paper.

\section{Acknowledgments}

The authors wish to acknowledge the financial support from the University of Malaya (UM) and Ministry of Education of Malaysia (Grant nos. FP027/2012A, PG078/2013B, and UM.C/625/1/HIR /MOHE/ENG/55).

\section{References}

[1] D. K. Babajee, M. Z. Dauhoo, M. T. Darvishi, A. Karami, and A. Barati, "Analysis of two Chebyshev-like third order methods free from second derivatives for solving systems of nonlinear equations," Journal of Computational and Applied Mathematics, vol. 233, no. 8, pp. 2002-2012, 2010.

[2] C. Chun, "A simply constructed third-order modifications of Newton's method," Journal of Computational and Applied Mathematics, vol. 219, no. 1, pp. 81-89, 2008.

[3] C. Chun and Y. Ham, "Some fourth-order modifications of Newton's method," Applied Mathematics and Computation, vol. 197, no. 2, pp. 654-658, 2008.

[4] J. R. Sharma, "A composite third order Newton-Steffensen method for solving nonlinear equations," Applied Mathematics and Computation, vol. 169, no. 1, pp. 242-246, 2005.

[5] J. C. Mason and D. C. Handscomb, Chebyshev Polynomials, Chapman \& Hall/CRC Press, 2003.

[6] J. Kou, Y. Li, and X. Wang, "A modification of Newton method with third-order convergence," Applied Mathematics and Computation, vol. 181, no. 2, pp. 1106-1111, 2006.

[7] D. Li, P. Liu, and J. Kou, "An improvement of Chebyshev-Halley methods free from second derivative," Applied Mathematics and Computation, vol. 235, no. 25, pp. 221-225, 2014.

[8] W. Gautschi, Numerical Analysis, Springer, Berlin, Germany, 2011.

[9] R. Askey, Orthogonal Polynomials and Special Functions, Society for Industrial and Applied Mathematics, Philadelphia, Pa, USA, 1975.

[10] S. H. Mahdavi and H. Abdul Razak, "A wavelet-based approach for vibration analysis of framed structures," Applied Mathematics and Computation, vol. 220, pp. 414-428, 2013.

[11] S. H. Mahdavi and S. Shojaee, "Optimum time history analysis of SDOF structures using free scale of Haar wavelet," Structural Engineering and Mechanics, vol. 45, no. 1, pp. 95-110, 2013.

[12] W. M. Abd-Elhameed, E. H. Doha, and Y. H. Youssri, "New wavelets collocation method for solving second-order multipoint boundary value problems using Chebyshev polynomials of third and fourth kinds," Abstract and Applied Analysis, vol. 2013, Article ID 542839, 9 pages, 2013.

[13] W. Swaidan and A. Hussin, "Feedback control method using Haar wavelet operational matrices for solving optimal control problems," Abstract and Applied Analysis, vol. 2013, Article ID 240352, 8 pages, 2013.

[14] E. Babolian and F. Fattahzadeh, "Numerical solution of differential equations by using Chebyshev wavelet operational matrix of integration," Applied Mathematics and Computation, vol. 188, no. 1, pp. 417-426, 2007.
[15] S. H. Mahdavi and H. A. Razak, "A comparative study on optimal structural dynamics using wavelet functions," Mathematical Problems in Engineering. In press.

[16] S. H. Mahdavi and H. A. Razak, "An indirect time integration scheme for dynamic analysis of space structures using wavelet functions," Journal of Engineering Mechanics (ASCE), In Press.

[17] Z. Li and Y. Wang, "Second Chebyshev wavelet operational matrix of integration and its application in the calculus of variations," International Journal of Computer Mathematics, vol. 90, no. 11, pp. 2338-2352, 2013.

[18] M. A. Crisfield, Non-Linear Finite Element Analysis of Solids and Structures: Advanced Topics, John Wiley \& Sons, New York, NY, USA, 1997. 


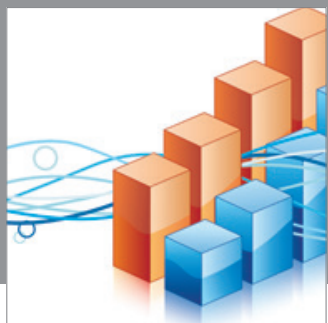

Advances in

Operations Research

mansans

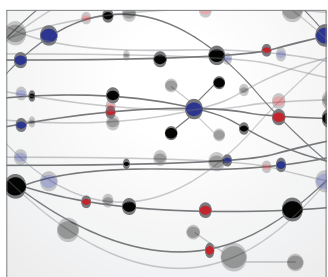

The Scientific World Journal
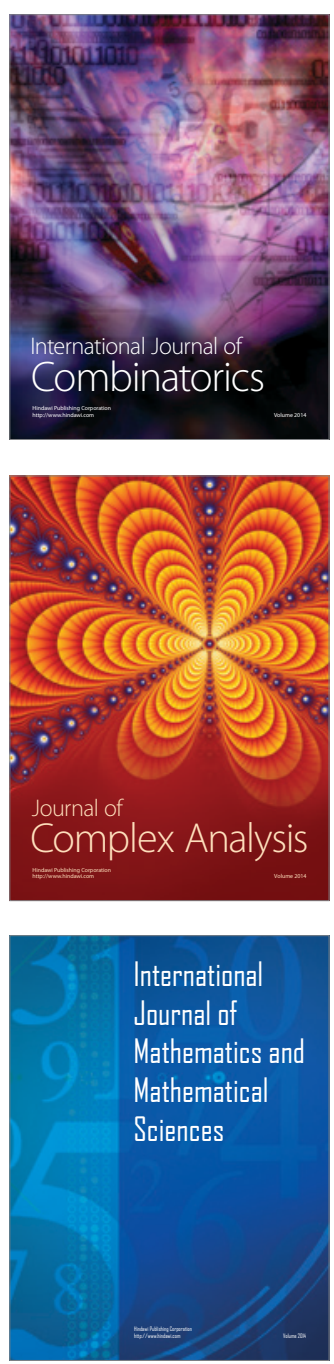
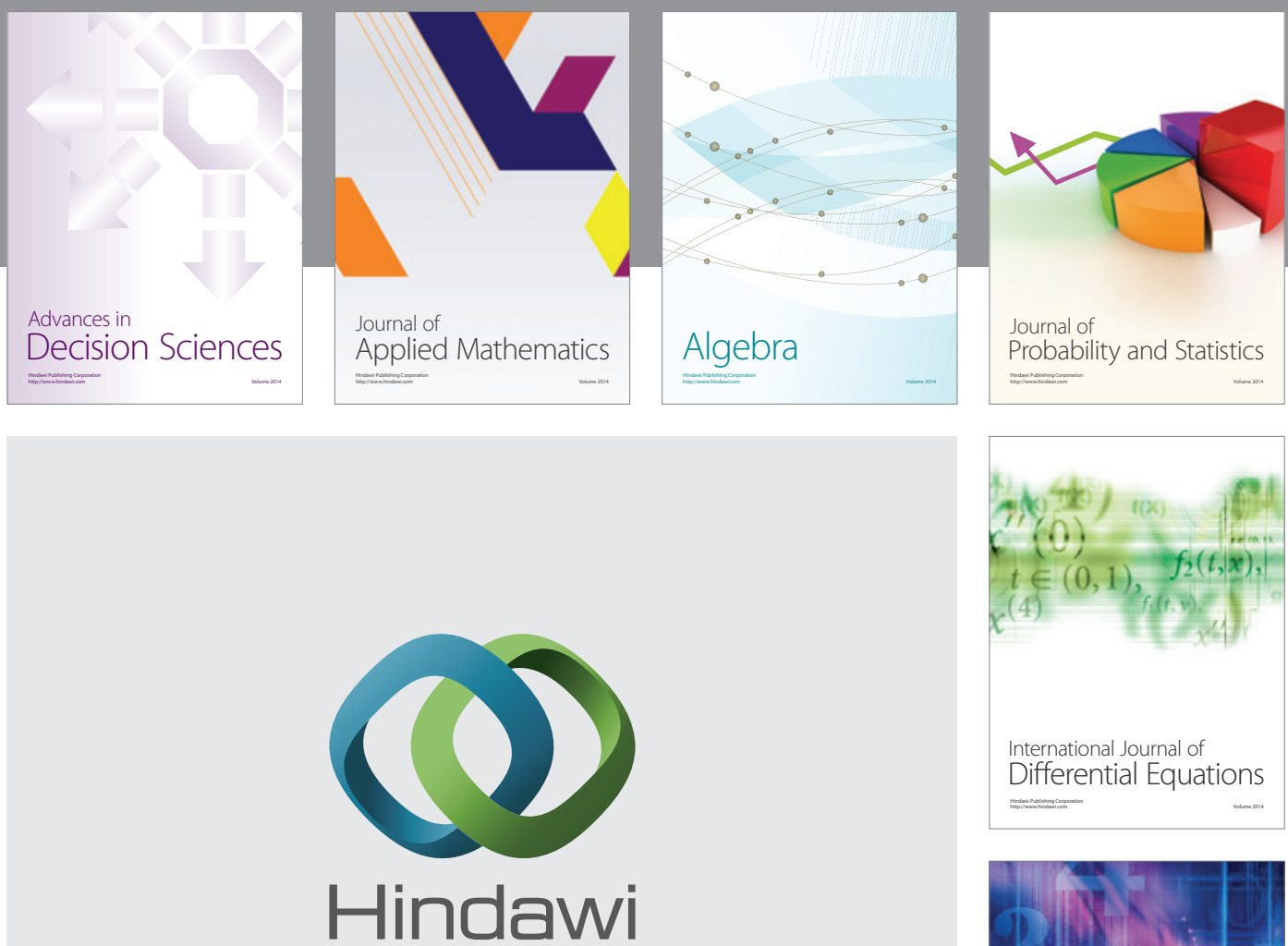

Submit your manuscripts at http://www.hindawi.com
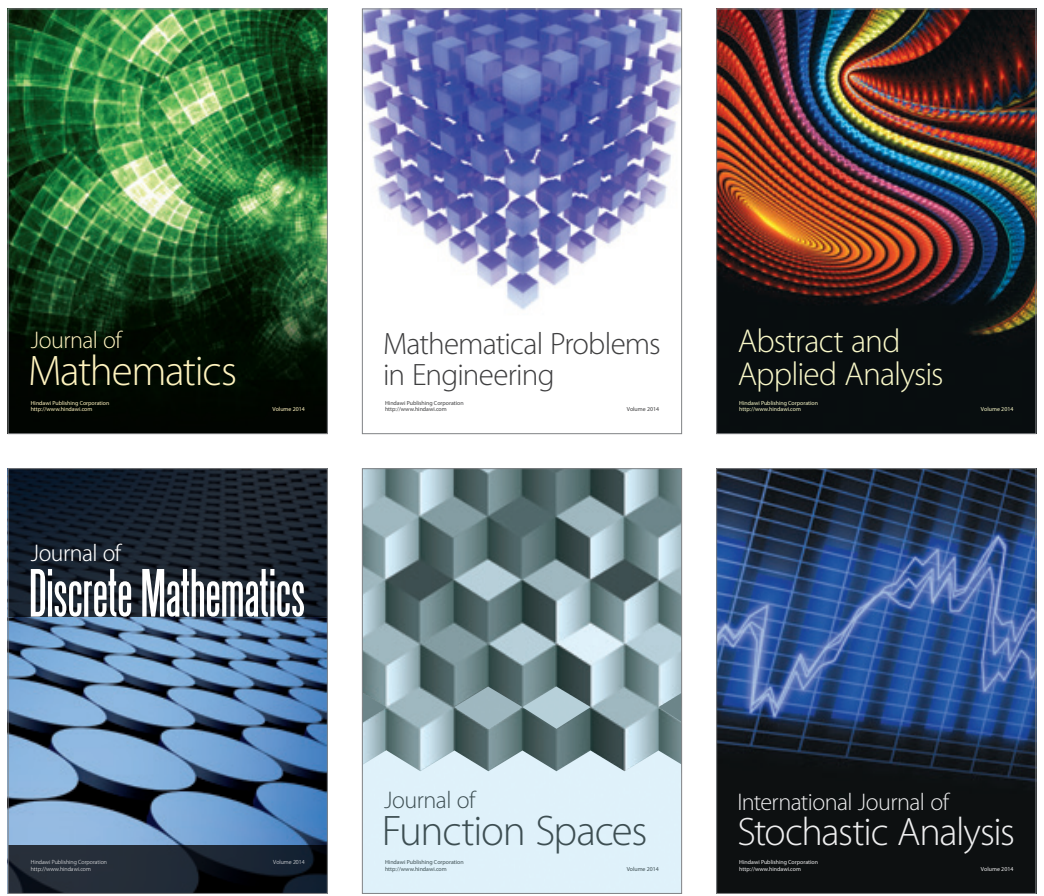

Journal of

Function Spaces

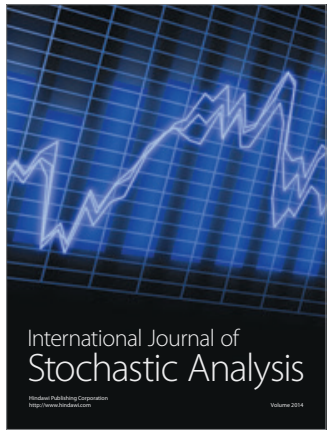

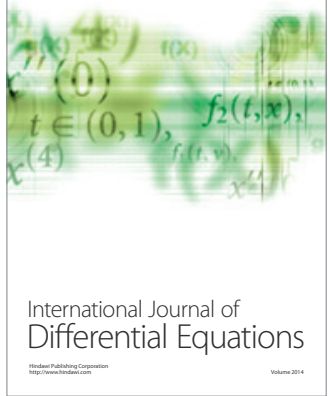
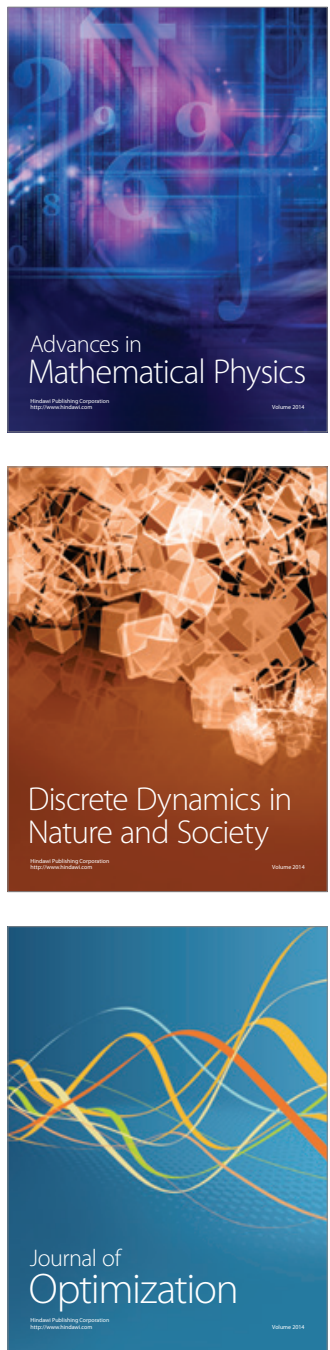